\title{
Isolation of the Buchnera aphidicola flagellum basal body from the Buchnera membrane
}

Matthew J. Schepers ${ }^{1}$, James N. Yelland ${ }^{1}$, Nancy A. Moran ${ }^{2 *}$, David W. Taylor ${ }^{1,3-5 *}$

${ }^{1}$ Institute for Cell and Molecular Biology, University of Texas at Austin, Austin, TX, 78712

${ }^{2}$ Department of Integrative Biology, University of Texas at Austin, Austin, TX, 78712

${ }^{3}$ Departmnet of Molecular Biosciences, University of Texas at Austin, Austin, TX, 78712

${ }^{4}$ Center for Systems and Synthetic Biology, University of Texas at Austin, Austin, TX, 78712

${ }^{5}$ LIVESTRONG Cancer Institute, Dell Medical School, Austin, TX, 78712

*Correspondence to: dtaylor@utexas.edu (D.W.T.); nancy.moran@austin.utexas.edu (N.A.M.) 


\begin{abstract}
Buchnera aphidicola is an intracellular bacterial symbiont of aphids and maintains a small genome of only $600 \mathrm{kbps}$. Buchnera is thought to maintain only genes relevant to the symbiosis with its aphid host. Curiously, the Buchnera genome contains gene clusters coding for flagellum basal body structural proteins and for flagellum type III export machinery. These structures have been shown to be highly expressed and present in large numbers on Buchnera cells. No recognizable pathogenicity factors or secreted proteins have been identified in the Buchnera genome, and the relevance of this protein complex to the symbiosis is unknown. Here, we show isolation of Buchnera flagella from the cellular membrane of Buchnera, confirming the enrichment of flagellum proteins relative to other proteins in the Buchnera proteome. This will facilitate studies of the structure and function of the Buchnera flagellum structure, and its role in this model symbiosis.
\end{abstract}




\section{Introduction}

Buchnera aphidicola is an obligate endosymbiont of aphid species worldwide ${ }^{1}$ and is a model for bacterial genome reduction, maintaining one of the smallest genomes yet discovered, only $600 \mathrm{kbps}^{2},{ }^{3}$. Though Buchnera has lost genes not essential for its symbiotic lifestyle ${ }^{2},{ }^{4},{ }^{5}$ it retains genes associated with amino acid biosynthesis, reflecting its participation in a nutritional symbiosis ${ }^{2},,, 7$. Though the exchange of amino acids and vitamins between the aphid host and Buchnera has been well-documented ${ }^{6},{ }^{8}, 9$, the molecular mechanism for how these metabolites cross Buchnera membranes is unknown: Buchnera maintains a small number of genes coding for membrane transport proteins, most of which are located at the inner membrane ${ }^{2},{ }^{10}$. The permeability of the Buchnera outer membrane remains a mystery, considering the paucity of annotated transporter genes in sequenced Buchnera genomes. Genes coding for proteins localizing to the outer membrane of Buchnera include small $\beta$-barrel aquaporins, which allow passive diffusion of small molecules, and flagellum basal body components ${ }^{2},{ }^{9},{ }^{10}$. Investigation into protein expression by these symbiotic partners has shown that flagellum basal body components are highly expressed by Buchnera ${ }^{11}$. Indeed, transmission electron microscopy images of Buchnera reveal flagellum basal bodies studded all over the bacterial outer membrane ${ }^{12}$. Despite its abundance on the Buchnera cell surface, the role of this protein complex for maintaining the aphid-Buchnera symbiosis is unknown ${ }^{13}$.

Buchnera of the pea aphid (Acyrthosiphon pisum) maintains 26 genes coding for flagellum proteins in three discrete clusters. The maintained genes code for the structural proteins required for formation of a flagellum basal body, a partial flagellar hook, as well as the Type III cytoplasmic export proteins. Buchnera lineages vary in the set of flagellum genes retained (Supplementary Table 1), but all have lost genes encoding the flagellin and motor proteins ${ }^{14}$, indicating a functional shift away from cell motility. The bacterial flagellum structure is an evolutionary homologue to the injectisome (Type III secretion system, or T3SS), a macromolecular protein complex used to 
deliver secrete effector proteins, often to a eukaryotic host ${ }^{15},{ }^{16},{ }^{17}$. Flagellum assembly occurs in a stepwise, sequential manner beginning from the bacterial cytoplasm, identical to the $\mathrm{T} \mathrm{SS}^{18},{ }^{19},{ }^{20}$. Buchnera maintains genes coding for the proteins required for a functional T3SS ${ }^{2},{ }^{12}$, as shown in studies of Yersinia ${ }^{21}$, and Salmonella ${ }^{22}{ }^{23}$. Gram-negative bacteria have also been shown to export proteins through a flagellum basal body $21,24,25$. The bacterial flagellum could be repurposed to serve a novel function for the aphid-Buchnera symbiosis. The basal body could serve as a type III protein exporter to secrete proteins to signal to the aphid host or as an surface signal molecule for host recognition during infection of new aphid embryos. Here, we present a procedure for isolation of flagellum basal body complexes adapted for an endosymbiont ${ }^{26}$, allowing for removal of these structures directly from Buchnera and enrichment of flagellum basal body complexes after isolation. This procedure will enable further characterization of the basal bodies and their modifications for a role in symbiosis.

\section{Results}

Isolation of hook basal bodies from Buchnera

Purification of the complex was initially assessed at multiple timepoints along the procedure. Samples were taken of initial Buchnera cell lysate, lysate after raising the $\mathrm{pH}$ to 10 , protein suspension after the first $5000 \mathrm{~g}$ spin, the third $5000 \mathrm{~g}$ spin, and finally after the $30,000 \mathrm{~g}$ spin and overnight incubation in TET buffer. SDS-PAGE showed sixteen bands were present after the staining procedure and their sizes corresponded to those of constituent proteins of the Buchnera flagellum basal body (Supplemental figure 1). Protein samples were extracted from the gel and subjected to mass spectrometry analysis.

\section{Mass spectrometry analysis of isolated basal bodies}

Protein ID LC-MS/MS spectral counts were provided by the University of Texas at Austin Proteomics Core Facility. We compared our samples to proteomic datasets from homogenized whole aphids, and from bacteriocytes purified from pea aphids ${ }^{11}$. Buchnera flagellum proteins 
were highly enriched by our isolation procedure, especially FliF, Flgl, FlgE, FlhA, and FlgF (Figure 1.). These results indicate that all but two flagellum proteins present in the mass spectrometry samples were enriched during the isolation procedure: structural proteins FilE, FliF, Flgl, FlgE, $\mathrm{FlgF}$, and $\mathrm{FlgH}$ were enriched threefold or more from the start to the finish of the procedure. FlgB, FlgC, FlgG, FliG, FliH, and Flil were enriched, though not to the extent of the other structural proteins. Type III secretion proteins FlhA and FliP were shown to be enriched by this procedure (Figure 2., Supplemental figure 2.). The widespread enrichment of Buchnera flagellum proteins indicates that our adapted procedure for isolating macromolecular protein complexes from the membranes of endosymbiotic bacteria was successful. Only flagellum proteins FlgK and FliN were reduced by the isolation procedure, perhaps because of their localization to the periphery of the flagellum.

\section{Basal bodies resemble top hats via electron microscopy}

We analyzed the isolated basal bodies by negative stain electron microscopy. While raw micrographs showed heterogenous particles, likely due to disassembly of the complex, detergent micelles, and contaminating proteins, there were several particles that appeared regularly. These single particles resembled a top hat with both rod and ring-shaped features (Figure 3), similar in size and shape to those observed in TEM images of whole Buchnera cells ${ }^{12}$.

\section{Discussion}

Here, we demonstrate a procedure for isolating macromolecular protein complexes from Buchnera aphidicola, an obligate endosymbiotic bacterium that cannot be cultured or genetically manipulated. Identifying the changes in these complexes could elucidate how Buchnera's adaptation over millions of years to a mutualistic lifestyle has affected its proteome.

As Buchnera is not motile and is confined to host-derived "symbiosomal" vesicles inside bacteriocytes ${ }^{28}{ }^{29}$, the retention and expression of these partial flagella indicates that they have become repurposed. These complexes have previously been hypothesized to be acting as type 
III secretion systems for provisioning peptides or signal factors to the aphid host ${ }^{13}$. Indeed, the proteins retained in the Buchnera flagellum constitute the structural proteins and machinery required for a functional type III secretion system ${ }^{21}$. Transcriptome analyses of pea aphid lines with different Buchnera titers reveal differences in expression of flagellar genes ${ }^{30}$. In aphid lines that harbor relatively low numbers of Buchnera, the endosymbionts have elevated relative expression of mRNA associated with flagellar secretion genes ( $f l i P, f l i Q$, and fliR), while Buchnera in aphid lines with high Buchnera numbers had elevated expression of genes for flagellum structural proteins ${ }^{30}$

Though heavily expressed in Buchnera of pea aphids, components of the flagellum basal body are not maintained equally among lineages of Buchnera of different aphid species based on available genomic sequences ${ }^{14}$ (Supplementary Table 1). Genes coding for proteins associated with type III secretion activity ( $f(h A, f l h B, f l i P, f l i Q$, and fliR) and basal body structural proteins (fliE, $f l i F, f l g B, f l g C, f l g F, f l g G$, and $f l g H)$ are well maintained across Buchnera lineages, but genes coding for hook proteins (flgD, flgE, and $f(g K)$ and the flagellum-specific ATPase (flil) are frequently shed. A more extreme example is the Buchnera strain harbored by aphids of genus Stegophylla: having the smallest sequenced Buchnera genome discovered thus far (412 kbps), these Buchnera have completely lost genes associated with flagellum structure and Type III secretion activity. In all but the most extreme examples, the Buchnera flagellum is well maintained, pointing to a continuing role for this complex for this ancient symbiosis.

Buchnera's tiny genome contains no known pathogenicity proteins or proteins previously associated with type III export ${ }^{2},{ }^{31}$. Potentially, Buchnera flagellum basal bodies may instead serve as surface signals for recognition by the host. Vertical transfer of Buchnera from mother to daughter aphids shows naked Buchnera cells being exocytosed from maternal bacteriocytes and moving in aphid haemolymph to infect a nearby specialized syncytial cell of stage 7 embryos ${ }^{32}$. The purpose of the flagellum in the context of Buchnera's symbiotic lifestyle remains unknown. 
Further inquiry into this protein complex could reveal how the repurposing of a motility organelle facilitates this ancient and obligate symbiosis.

\section{Methods}

\section{Buchnera extraction from aphids}

Pea aphids (Acrythosiphon pisum strain LSR1) were placed as all-female clones on Fava bean (Vicia faba) seedlings on $16 \mathrm{~h} / 8 \mathrm{~h}$ light/dark cycles at $20^{\circ} \mathrm{C}$. Once reaching adulthood, apterous adults were raised on Fava bean plants on 16h/8h light cycles and allowed to reproduce. After seven days, all aphids (fourth-instar larvae, typically amounting to $5 \mathrm{~g}$ ) were removed from the Fava bean plants. Aphids were weighed and surface-sterilized in $0.5 \%$ bleach solution, then rinsed twice in Ultrapure water (MilliporeSigma), each 30 seconds. Aphids were gently ground in a mortar and pestle in $40 \mathrm{~mL}$ sterile Buffer A (25mM KCl (Sigma-Aldrich), 35mM Tris base (SigmaAldrich), $10 \mathrm{mM} \mathrm{MgCl}_{2}$ (Sigma-Aldrich), 250mM anhydrous EDTA (Sigma-Aldrich), and 500mM Sucrose (Sigma-Aldrich) at $\mathrm{pH}$ 7.5). Aphid homogenate was vacuum filtered to $100 \mu \mathrm{m}$, then centrifuged at $1500 \mathrm{~g}$ for 10 minutes at $4 \mathrm{C}$. Supernatant was discarded, and the resulting pellet was resuspended in $20 \mathrm{~mL}$ Buffer $A$ and vacuum-filtered three times from $20 \mu \mathrm{m}$, to $10 \mu \mathrm{m}$, and finally to $5 \mu \mathrm{m}$. The resulting filtrate was spun at $1500 \mathrm{~g}$ for $30 \mathrm{~m}$ at $4 \mathrm{C}$ and supernatant discarded. The resulting pellet was resuspended in $10 \mathrm{~mL}$ Sucrose solution (300mM sucrose (Sigma-Aldrich) and $100 \mathrm{mM}$ Tris base (Sigma-Aldrich) then checked on a brightfield microscope for intact Buchnera cells. Buchnera cells remain alive while at $4 \mathrm{C}$ for a maximum of $24 \mathrm{~h}$.

\section{Isolation of flagellum basal bodies from Buchnera cells}

Buchnera was incubated with gentle spinning on ice with egg white lysozyme $(0.1 \mathrm{mg} / \mathrm{mL}$, Sigma-Aldrich) for 30m. 100mM Anhydrous EDTA solution, pH 7.5 (Sigma-Aldrich) was added to final concentration $10 \mathrm{mM}$. The pellet was taken off ice, and gradually raised to room temperature with gentle spinning for $30 \mathrm{~m}$. Triton $\mathrm{X}-100$ (Acros Organics) was added to $1 \% \mathrm{w} / \mathrm{v}$, along with 1mg/mL RNase-free DNase I (Bovine Pancreas, Sigma-Alrich) and allowed to stir for 1/2 hour. After incubation, cell lysate was kept at $4 \mathrm{C}$ or on ice until use. The lysate was raised to $\mathrm{pH} 10$ 
using $1 \mathrm{~N} \mathrm{NaOH}$ (Macron Fine Chemicals) to attempt to denature host and bacterial cytoplasmic proteins. The solution was spun at $5000 \mathrm{~g}$ for $10 \mathrm{~m}$ at $4 \mathrm{C}$ three times, each time decanting the supernatant to a new tube. After three spins, the supernatant was transferred to a Nalgene Oak Ridge polyallomer centrifuge tube (Thermo-Fisher) and spun at $30,000 \mathrm{~g}$ for $1 \mathrm{~h}$ at $4 \mathrm{C}$. Supernatant was gently decanted and pellet covered with TET buffer $(10 \mathrm{mM}$ Tris- $\mathrm{HCl}, 5 \mathrm{mM}$ EDTA, $0.1 \% \mathrm{X}$ 100, $\mathrm{pH} 8.0$ ) and left overnight at $4 \mathrm{C}$ to soften and dissolve.

Submission of protein for mass spectrometry

Solubilized protein concentration was determined using an Eppendorf Biophotometer. $1.5 \mathrm{mg}$ protein was run on premade $4-12 \%$ Tris-Glycine SDS-PAGE gels (Thermo-Fisher) at 120V for $10 \mathrm{~m}$. Gels were stained in Coomassie Brilliant Blue (Bio-Rad) for 30m, then destained in $20 \%$ Acetic acid (Thermo-Fisher) for $30 \mathrm{~m}$. Gel bands corresponding to the step in the procedure sampled ("Lysate," "pH 10," "Spin 1," "Spin 3," "Final”) were cut out and submitted to the University of Texas at Austin CBRS Biological Mass Spectrometry Facility for LC-MS/MS using a Dionex Ultimate 3000 RSLCnano LC coupled to a Thermo Orbitrap Fusion (Thermo-Fisher). Samples were submitted in $50 \mathrm{~mL}$ destain with Buchnera aphidicola str. APS provided as the reference organism (ASM960v1). Prior to HPLC separation, peptides were desalted using Millipore U-C18

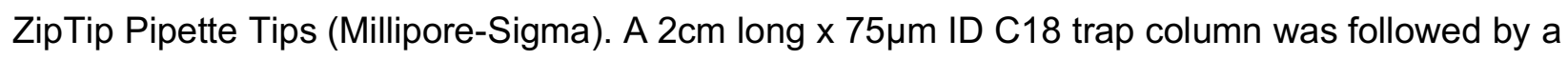
$25 \mathrm{~cm}$ long $\times 75 \mu \mathrm{m}$ analytical columns packed with C18 3um material (Thermo Acclaim PepMap 100, Thermo-Fisher) running a gradient from 5-35\%. The FT-MS resolution was set to 120,000, with an MS/MS cycle time of 3 seconds and acquisition in HCD ion trap mode. Raw data was processed using SEQUEST HT embedded in Proteome Discoverer (Thermo-Fisher). Scaffold 4 (Proteome software) was used for validation of peptide and protein IDs.

\section{EM and data collection}

Protein from the final step of this procedure was stained using 3\% Uranyl Acetate on a 400-mesh continuous carbon grid. Images were acquired using an FEl Talos transmission 
electron microscope operating at $200 \mathrm{kV}$, with 1.25 second exposures, a dose rate of $19 \mathrm{e}^{-} \AA^{-2}$, and a nominal magnification of $57,000 X$.

\section{Whole aphid proteomic samples}

For controls, proteomes were profiled for whole aphids, including both Buchnera and aphid cells. Aphids were mixed-aged populations grown at $20^{\circ} \mathrm{C}$ in 30 cup cages and pooled into three replicate samples. Aphids were washed and homogenized in buffer as described above. The homogenate was centrifuged at $4000 \mathrm{~g}$ for $15 \mathrm{~min}$ at $4^{\circ} \mathrm{C}$, Supernatant was removed, and pellet was suspended with $2 \%$ SDS, $0.1 \mathrm{M}$ Tris- $\mathrm{HCl}, 0.1 \mathrm{M} \mathrm{DTT}$ at $100^{\circ} \mathrm{C}$ for $10 \mathrm{~min}$, then centrifuged at $14,000 \mathrm{~g}$ for $20 \mathrm{~min}$ at $4 \mathrm{C}$ to remove non-soluble material after adding same volume of $8 \mathrm{M}$ Urea. Protein concentration was determined on an Eppendorf BioPhotometer. 5mg total protein was run on a Bis-Tris gel for less than $1 \mathrm{~cm}$, and the band was excised and and sent to the UT Proteomics Core for LC-MS/MS protein ID. Protein ID methods were identical as detailed above.

Author contributions

M.J.S. raised aphids and prepared Buchnera protein extracts. J.N.Y. performed electron microscopy. N.A.M. and D.W.T. analyzed data and supervised and secured funding for this work. All authors reviewed the final manuscript.

\section{Acknowledgements}

We thank Eric Verbeke, Jack Bravo, and Evan Schwartz for their advice and ideas for isolating and imaging proteins from native cells; Julie Perreau, Margaret Steele, and Serena Zhao for creating a space in which ideas and techniques could be shared freely; Kim Hammond for help with aphid raising and organization. This work was supported by the National Science Foundation 1551092 (to N.A.M), a Welch Foundation grant F-1938 
(to D.W.T.), National Institute of General Medical Sciences (NIGMS) of the National Institutes of Health (NIH) R35GM138348 (to D.W.T.), Army Research Office Grant W911NF-15-1-0120 (to D.W.T.), and a Robert J. Kleberg, Jr. and Helen C. Kleberg Foundation Medical Research Award (to D.W.T.). D.W.T is a CPRIT Scholar supported by the Cancer Prevention and Research Institute of Texas (RR160088) and an Army Young Investigator supported by the Army Research Office (W911NF-19-1-0021).

Competing interests

The authors declare no competing interests. 


\section{Figures}

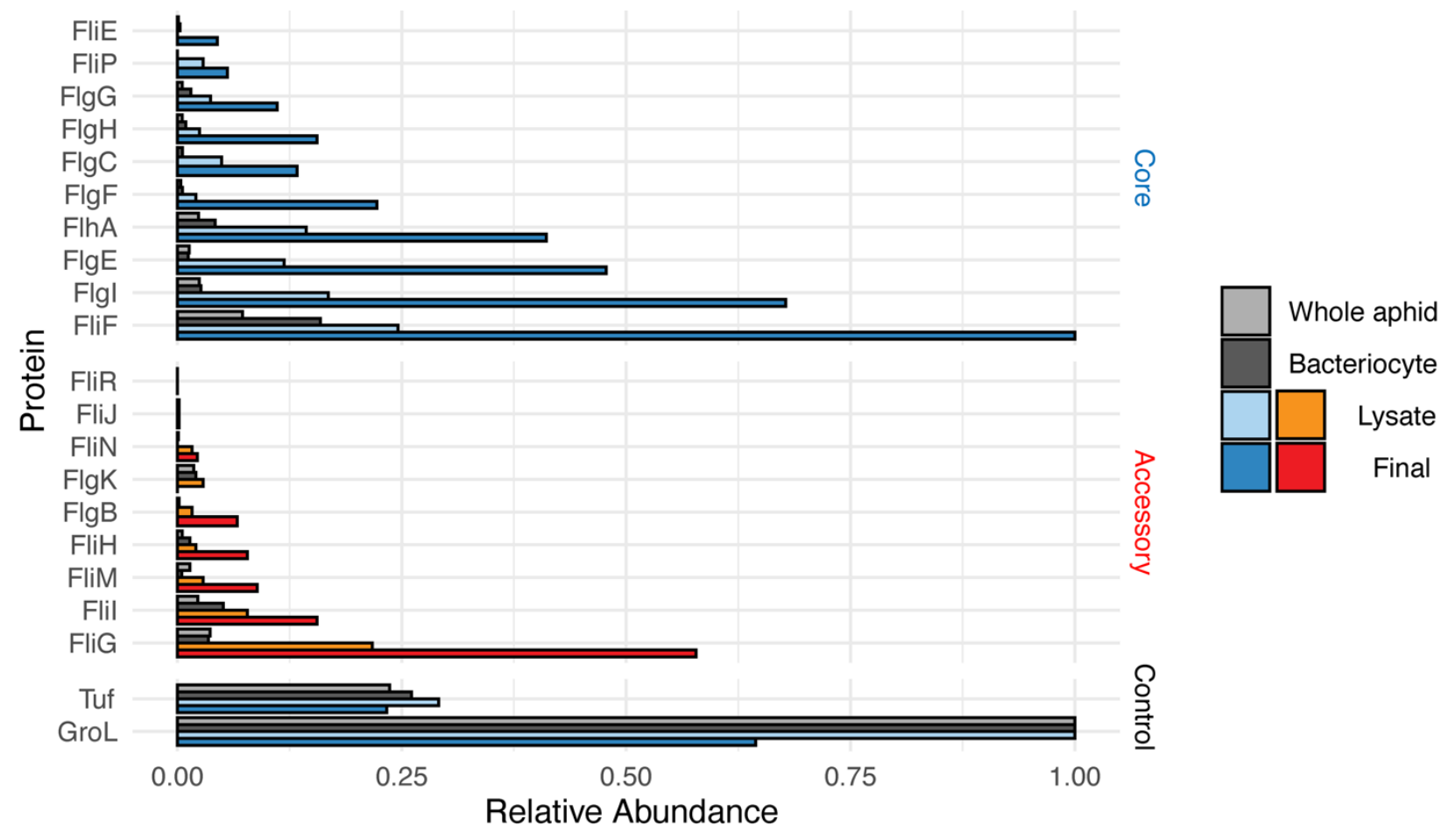

Figure 1: Barplot showing flagellum protein enrichment before (Lysate) and after (Final) the isolation procedure compared to proteomic datasets generated with whole aphids and dissected bacteriocytes. Blue indicates "core" proteins required for secretion activity and red indicates accessory proteins maintained by Buchnera aphidicola in pea aphids. 


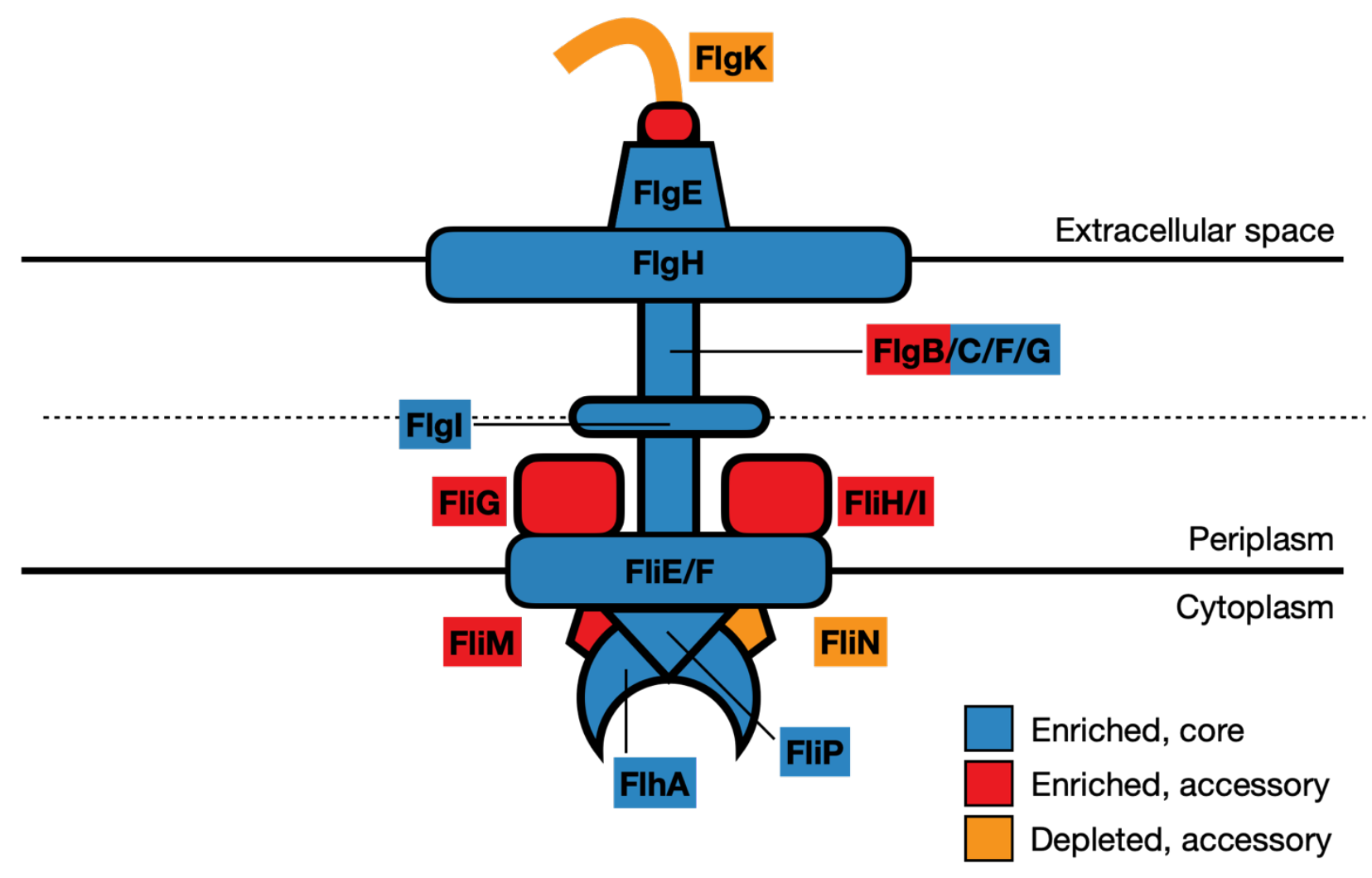

Figure 2: Cartoon diagram of the reduced Buchnera aphidicola (pea aphid) flagellum. Colors indicate enrichment status of individual proteins at the final step of the procedure, corresponding to Figure 1. 

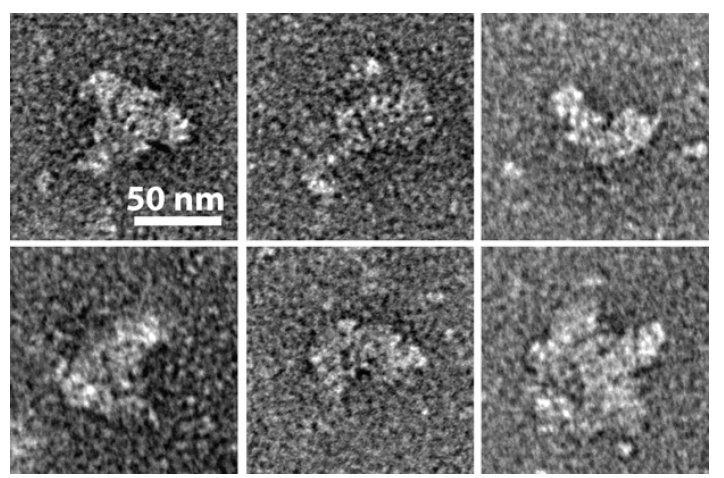

Figure 3: Single particles of Buchnera flagellum complexes after the isolation procedure. Scale bars represent $50 \mathrm{~nm}$. 


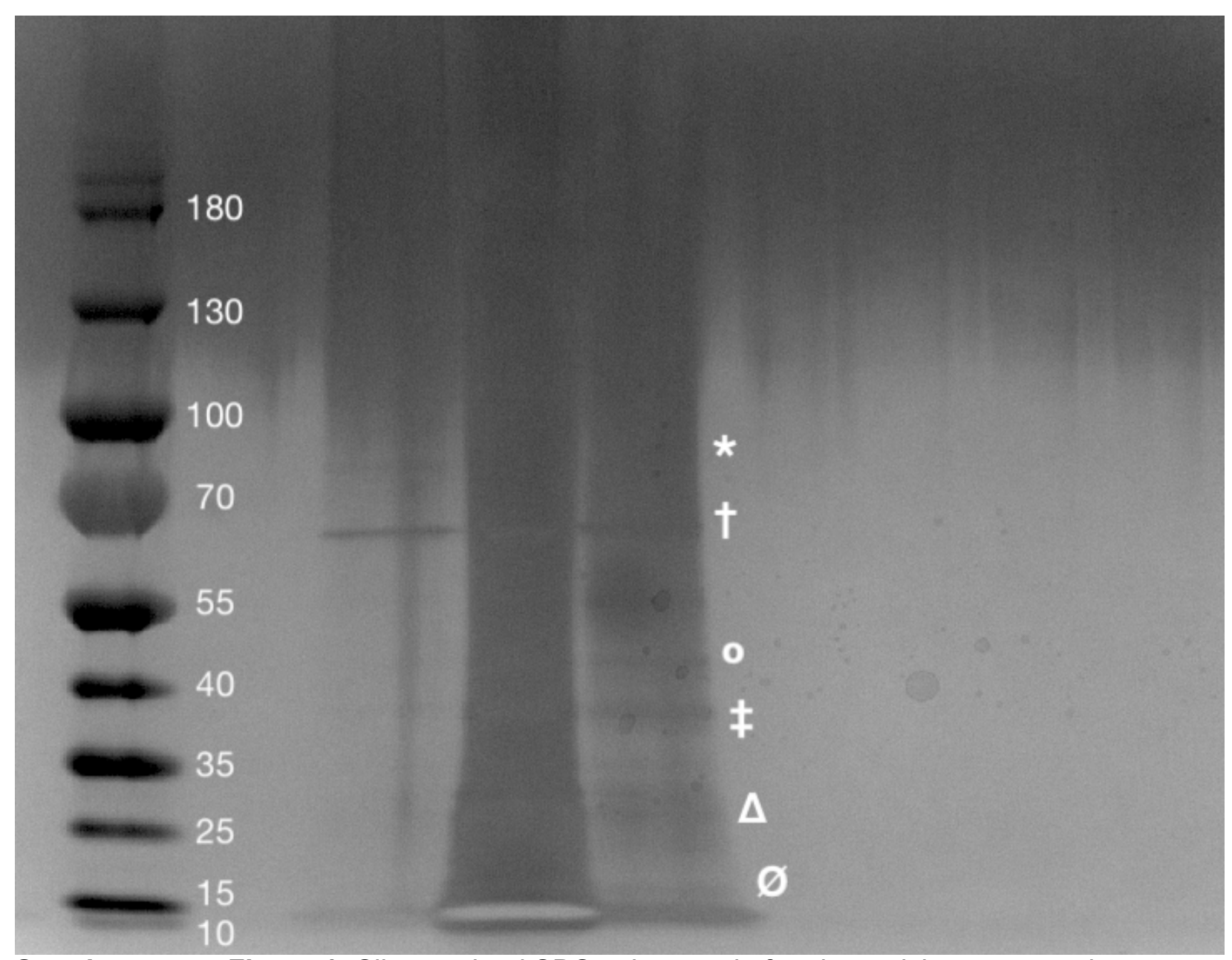

Supplementary Figure 1: Silver stained SDS gel created after the enrichment procedure was performed. The first lane is taken directly from the enrichment preparation after overnight incubation with TET buffer. The second lane is after concentrating the enriched proteins to 1 $\mathrm{mg} / \mathrm{mL}$. The third lane is concentrated protein diluted to $0.5 \mathrm{mg} / \mathrm{mL}$. Ladder values represent molecular weight in $\mathrm{kDa}$. Symbols correspond to flagellar protein molecular weight:

* corresponds to FlhA $(78 \mathrm{kDa})$.

† corresponds to FliF ( $63 \mathrm{kDa})$ and FlgK $(63 \mathrm{kDa})$.

${ }^{\circ}$ corresponds to FlgE (45 kDa), FliP (43kDa), and Flgl (41 kDa).

$\ddagger$ corresponds to FliG $(38 \mathrm{kDa})$ and FliM $(37 \mathrm{kDa})$.

$\Delta$ corresponds to FlgG (28 kDa), FlgF (28 kDa), FlgH $(26 \mathrm{kDa})$, and FliH (26kDa).

$\varnothing$ corresponds to FlgB (16 kDa), FliN (15 kDa), FlgC (15 kDa), and FliE (11 kDa). 


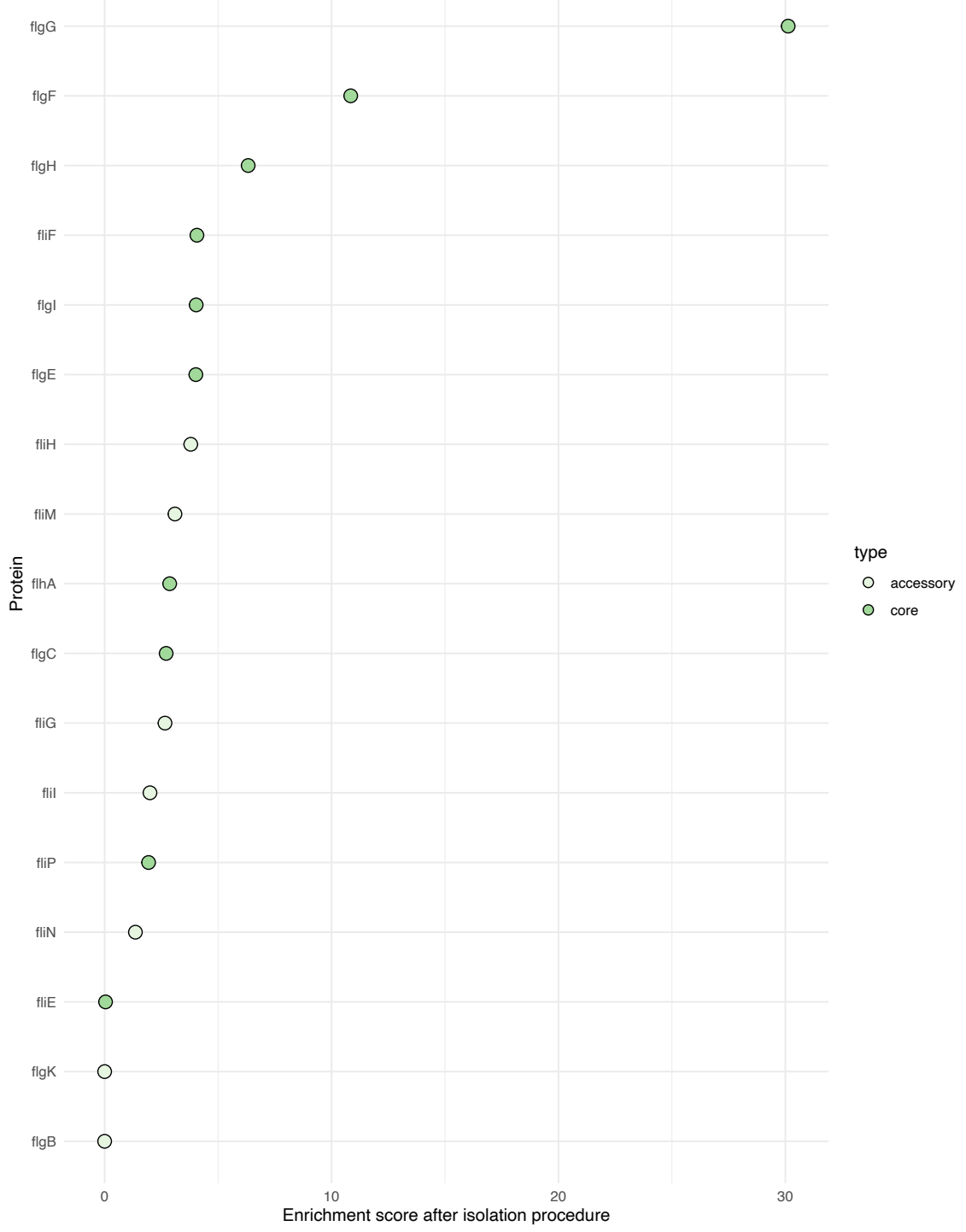

Supplementary Figure 2: Dotplot of Buchnera aphidicola flagellum proteins found after LC/MSMS analysis. The enrichment score for each protein is indicated on the $x$ axis. Enrichment scores are calculated by dividing unique spectral counts for each protein in the final step by each protein present in the cell lysate. Core flagellum proteins (defined by proteins required for type III secretion activity and flagellum structure) are filled in green, accessory proteins are filled in white. 


\section{References}

1. Munson, M.A., Baumann, P., Kinsey, M.G. Buchnera gen. nov. and Buchnera aphidicola sp. nov., a taxon consisting of the mycetocyte-associated, primary endosymbionts of aphids. Int. J. Syst. Bacteriol. 1991; 41:566-568

2. Shigenobu, S., Watanabe, H., Hattori, M., Sakaki, Y. \& Ishikawa, H. Genome sequence of the endocellular bacterial symbiont of aphids Buchnera sp. APS. Nature 407, 81-86 (2000).

3. Moran, N. A. \& Bennett, G. M. The tiniest tiny genomes. Annu Rev Microbiol 68, 195-215 (2014).

4. Tamas, I. et al. 50 million years of genomic stasis in endosymbiotic bacteria. Science 296, 2376-2379 (2002).

5. Wernegreen, J. J. Genome evolution in bacterial endosymbionts of insects. Nature Reviews Genetics 3, 850-861 (2002).

6. Douglas, A. E. Nutritional interactions in insect-microbial symbioses: aphids and their symbiotic bacteria Buchnera. Annu Rev Entomol 43, 17-37 (1998).

7. Akman Gündüz, E. \& Douglas, A. E. Symbiotic bacteria enable insect to use a nutritionally inadequate diet. Proc Biol Sci 276, 987-991 (2009).

8. Nakabachi, A. \& Ishikawa, H. Provision of riboflavin to the host aphid, Acyrthosiphon pisum, by endosymbiotic bacteria, Buchnera. J Insect Physiol 45, 1-6 (1999).

9. Charles, H., Calevro, F., Vinuelas, J., Fayard, J. M. \& Rahbe, Y. Codon usage bias and tRNA over-expression in Buchnera aphidicola after aromatic amino acid nutritional stress on its host Acyrthosiphon pisum. Nucleic Acids Res 34, 4583-4592 (2006).

10. Charles, $\mathrm{H}$. et al. A genomic reappraisal of symbiotic function in the aphid/Buchnera symbiosis: reduced transporter sets and variable membrane organisations. PLoS One 6, e29096 (2011).

11. Poliakov, A. et al. Large-scale label-free quantitative proteomics of the pea aphidBuchnera symbiosis. Mol Cell Proteomics 10, M110.007039 (2011).

12. Maezawa, K. et al. Hundreds of flagellar basal bodies cover the cell surface of the endosymbiotic bacterium Buchnera aphidicola sp. strain APS. J Bacteriol 188, 6539-6543 (2006).

13. Denise, R., Abby, S. S. \& Rocha, E. P. C. The Evolution of Protein Secretion Systems by Co-option and Tinkering of Cellular Machineries. Trends in Microbiology (2020).

14. Chong, R. A., Park, H. \& Moran, N. A. Genome evolution of the obligate endosymbiont Buchnera aphidicola. Mol Biol Evol (2019).

15. Cornelis, G. R. \& Van Gijsegem, F. Assembly and function of type III secretory systems. Annu Rev Microbiol 54, 735-774 (2000).

16. Moya, A., Peretó, J., Gil, R. \& Latorre, A. Learning how to live together: genomic insights into prokaryote-animal symbioses. Nat Rev Genet 9, 218-229 (2008).

17. Abby, S. S. \& Rocha, E. P. The non-flagellar type III secretion system evolved from the bacterial flagellum and diversified into host-cell adapted systems. PLoS Genet 8, e1002983 (2012).

18. Marlovits, T. C. et al. Structural insights into the assembly of the type III secretion needle complex. Science 306, 1040-1042 (2004).

19. Liu, R. \& Ochman, H. Stepwise formation of the bacterial flagellar system. Proc Natl Acad Sci U S A 104, 7116-7121 (2007).

20. Ince, D., Sutterwala, F. S. \& Yahr, T. L. Secretion of Flagellar Proteins by the Pseudomonas aeruginosa Type III Secretion-Injectisome System. Journal of Bacteriology 197, 2003-2011 (2015). 
21. Young, G. M., Schmiel, D. H. \& Miller, V. L. A new pathway for the secretion of virulence factors by bacteria: the flagellar export apparatus functions as a protein-secretion system. Proc Natl Acad Sci U S A 96, 6456-6461 (1999).

22. Irikura, V. M., Kihara, M., Yamaguchi, S., Sockett, H. \& Macnab, R. M. Salmonella typhimurium fliG and fliN mutations causing defects in assembly, rotation, and switching of the flagellar motor. J Bacteriol 175, 802-810 (1993).

23. Minamino, T. \& Macnab, R. M. Components of the Salmonella flagellar export apparatus and classification of export substrates. J Bacteriol 181, 1388-1394 (1999).

24. Konkel, M. E. et al. Secretion of virulence proteins from Campylobacter jejuni is dependent on a functional flagellar export apparatus. J Bacteriol 186, 3296-3303 (2004).

25. Scanlan, E., Yu, L., Maskell, D., Choudhary, J. \& Grant, A. A quantitative proteomic screen of the Campylobacter jejuni flagellar-dependent secretome. J Proteomics 152, 181-187 (2017).

26. López-Sánchez, M. J. et al. Evolutionary convergence and nitrogen metabolism in Blattabacterium strain Bge, primary endosymbiont of the cockroach Blattella germanica. PLoS Genet 5, e1000721 (2009).

27. Tegunov, D. \& Cramer, P. Real-time cryo-electron microscopy data preprocessing with Warp. Nat Methods 16, 1146-1152 (2019).

28. Braendle, C. et al. Developmental origin and evolution of bacteriocytes in the aphidBuchnera symbiosis. PLoS Biol 1, E21 (2003).

29. Miura, T. et al. A comparison of parthenogenetic and sexual embryogenesis of the pea aphid Acyrthosiphon pisum (Hemiptera: Aphidoidea). J Exp Zool B Mol Dev Evol 295, 5981 (2003).

30. Smith, T. E. \& Moran, N. A. Coordination of host and symbiont gene expression reveals a metabolic tug-of-war between aphids and Buchnera. Proc Natl Acad Sci U S A 117, 21132121 (2020).

31. Shimomura, S., Shigenobu, S., Morioka, M. \& Ishikawa, H. An experimental validation of orphan genes of Buchnera, a symbiont of aphids. Biochem Biophys Res Commun 292, 263-267 (2002).

32. Koga, R., Meng, X. Y., Tsuchida, T. \& Fukatsu, T. Cellular mechanism for selective vertical transmission of an obligate insect symbiont at the bacteriocyte-embryo interface. Proc Natl Acad Sci U S A 109, E1230-7 (2012). 